\title{
Diagnosis and management of depression
}

(Index words: depression, antidepressants, continuing medical education, diagnosis, management)

\section{Summary}

Depression is a disabling illness which decreases quality of life and productivity. The point prevalence of major depression ranges from $5 \%$ to $9 \%$ among adults in primary care settings. Both pharmacological and psychological treatment are effective in treating depression.

\section{Introduction}

Depression is experienced by people with a variety of health problems. It should be differentiated from sadness, which is a normal response to loss. Depression can present as a symptom of some other illness such as dementia, parkinsonism, schizophrenia or substance use, or it can be considered as a syndrome - depressive disorder. In depressive disorder there is a prolonged lowering of mood accompanied by cognitive and behavioural changes.

\section{Epidemiology}

Major depressive disorder is estimated to be the fourth leading cause of loss measured by Disability Adjusted Life Years (DALY), and ranks above ischaemic heart disease and cerebrovascular disease [1]. (The DALY combines the time lived with disability and the time lost due to premature mortality).

The lifetime risk for major depressive disorder is 10 $25 \%$ in women and $5-12 \%$ in men. It is significant that women are twice as likely as men to develop depression although the reasons for this difference are unclear.

Most depressed patients come to primary care settings and the point prevalence of major depression ranges from $5 \%$ to $10 \%$ among adults. Frequently patients with depression present with pain, non-specific musculoskeletal symptoms and medically unexplained symptoms. Many depressed patients have vague symptoms. In one study up to $70 \%$ of depressed patients reporting to a primary care physician gave only somatic symptoms [2]. Despite the high prevalence in primary care settings up to $50 \%$ of depressed patients go undiagnosed.

\section{Clinical features}

According to the ICD 10 (International Classification of Diseases) diagnostic guidelines, depressed mood, loss of interest and enjoyment, and increased fatigue are the most typical symptoms of depression. At least two of them together with at least two other symptoms listed in panel 1 should be present for at least 2 weeks for diagnosis.

\section{Panel 1. ICD-10 guidelines for diagnosing a depressive episode}

\section{Typical symptoms}

- Depressed mood

- Loss of interest and enjoyment of activities that are normally pleasurable

- Decreased energy or increased fatigue

\section{Other symptoms}
- Reduced concentration and attention
- Reduced self-esteem and self-confidence
- Ideas of guilt and unworthiness
- Bleak and pessimistic views of the future
- Ideas or acts of self-harm or suicide
- Disturbed sleep
- Diminished appetite

Mild depressive episode: at least 2 typical symptoms and 2 other symptoms

Moderate depressive episode: at least 2 typical symptoms and 3 (preferably 4 ) other symptoms

Severe depressive episode: all 3 typical symptoms and 4 other symptoms 


\section{Screening}

The key to diagnosing depression is a high degree of clinical suspicion. Many formal screening tools are available such as the Beck Depression Inventory and the General Health Questionnaire. These however, are difficult to use in a busy clinical setting. The United States Preventive Services Task Force recommends the screening of all adult patients with 2 key questions, which may be as effective as using longer instruments [3]. If the answer to either of these questions is 'yes' it is recommended that a clinical interview be conducted to exclude the diagnosis of major depressive disorder.

It is important to look for depression in patients with medically unexplained symptoms. Doctors generally tend to look only for physical causes in patients with poor sleep, poor appetite and lack of energy, vague joint or limb pain, despite these being common features of depression.

\section{Panel 2. United States Preventive Services Task Force screening questions for depression}

1. Over the past 2 weeks, have you felt down, depressed, or hopeless?

2. Over the past 2 weeks, have you felt little interest or pleasure in doing things you normally do?

\section{Treatment}

Both pharmacological and psychological treatment are effective in treating depression. A combination of psychological therapy and antidepressant medication produces better outcomes than antidepressant medication alone in moderate and severely depressed patients [4].

\section{Antidepressants}

Tricyclic antidepressants and monoamine oxidase inhibitors were introduced in the late 1950s, and are extremely effective, but they are limited in their usefulness because of side-effects and toxicity in overdose. The next class of antidepressants to be introduced were the specific serotonin reuptake inhibitors (SSRIs), fluoxetine, fluvoxamine, sertraline, citalopram, escitalopram and paroxetine. They are now considered as first line therapy in the treatment of depression.

Newer generations of antidepressants such as venlafaxine and duloxetine target multiple neurotransmitter systems (SNRIs - serotonin noradrenaline reuptake inhibitors). Other antidepressants available include nefazodone, trazadone, mirtazapine, reboxetine and bupropion. The reversible MAO inhibitor moclobemide

is safer when compared to the older non-reversible MAO inhibitors.

The SSRIs are recommended as first line treatment due to their effectiveness, less troublesome side-effects and lower toxicity in overdose. Drugs should be started at the minimum effective dose. These drugs have a long half-life and many can be administered once a day. It is important to tell patients that although side-effects may appear early a therapeutic response should not be expected until 10-14 days. If the response is poor after 2-6 weeks the dose can be increased [4].

Table 1. Effective dose ranges for antidepressants

\begin{tabular}{ll}
\hline Antidepressant drug & Effective dose \\
\hline Fluoxetine & $20-80 \mathrm{mg}$ \\
Paroxetine & $20-50 \mathrm{mg}$ \\
Fluvoxamine & $50-300 \mathrm{mg}$ \\
Sertraline & $50-200 \mathrm{mg}$ \\
Citalopram & $20-60 \mathrm{mg}$ \\
Venlafaxine XR & $75-225 \mathrm{mg}$ \\
Trazadone & $100-300 \mathrm{mg}$ \\
Mirtazapine & $15-45 \mathrm{mg}$ \\
Imipramine & $75-300 \mathrm{mg}$ \\
Amitriptyline & $75-250 \mathrm{mg}$ \\
\hline
\end{tabular}

In elderly patients medication should be started at lower doses and increased slowly depending on response.

Apart from pharmacological treatment, regular review and support in overcoming precipitating factors such as stress is necessary. Once symptoms are controlled treatment should be continued for a further 6 months and then gradually withdrawn [5].

\section{Psychological therapies}

Brief structured psychotherapies appear to be effective in the acute and long term management of mild and moderately severe depression. They are not suitable for severe depression. The three psychological therapies which have a proven effect in depressive disorder are cognitive behavioural therapy (CBT), interpersonal therapy and problem solving therapy. Cognitive behavioural therapy is aimed at changing the dysfunctional thinking patterns of depressed patients by repetitive challenge followed by behavioural changes. It has been shown that individuals prone to depressive episodes have dysfunctional thinking patterns even when they are in remission. It is possible that CBT reduces the 
future risk of depression. Interpersonal therapy (IPT) is aimed at improving the (usually impaired) interpersonal relationships of depressed patients. Both therapies could be practised by primary care physicians after a period of training. It has been demonstrated that CBT and IPT could be as effective as antidepressants in moderate depression.

\section{When to refer}

Most patients with depression can be managed in nonspecialised settings. However, patients with a risk of suicide and risk to life due to reduced food intake need in-patient treatment. Patients with a history of bipolar disorder should be referred to a psychiatrist as management of bipolar depression is often difficult. Patients who respond poorly to initial antidepressant treatment and those with psychotic depression (presence of hallucinations or delusions) too should be referred to a psychiatrist.

\section{Electroconvulsive therapy}

Electroconvulsive therapy is indicated when a rapid response is required, such as in severe depression where food intake is poor, or in those with high suicidal risk. Patients with psychotic depression respond well to ECT. Elderly patients often develop side-effects to antidepressant medication and may find it difficult to tolerate a therapeutic dose. Such patients benefit by ECT as do patients with resistant depression.

\section{Further reading}

1. Gelder MG, Cowen P, Harrison PJ. Shorter Oxford Textbook of Psychiatry. 5th ed. ed. Oxford: Oxford University Press; 2006.

2. Taylor D, Paton C, Kerwin R, South London and Maudsley NHS Trust, Oxleas NHS Foundation Trust. Prescribing guidelines. 9th ed. Taylor and Francis, 2007.

3. Stein DJ, Kupfer DJ, Schatzberg AF. The American Psychiatric Publishing Textbook of Mood Disorders, American Psychiatric Publishing Inc, 2005.

\section{References}

1. Murray CJL, Lopez AD. The global burden of disease: a comprehensive assessment of mortality and disability from diseases, injuries, and risk factors in 1990 and projected to 2020: summary. Harvard School of Public Health on behalf of the World Health Organization and the World Bank, 1996.

2. Gerber PD, Barrett JE, Barrett JA, Oxman TE, Manheimer E, et al. The relationship of presenting physical complaints to depressive symptoms in primary care patients. Journal of General Internal Medicine 1992; 7: $170-3$.

3. Whooley MA, Avins AL, Miranda J, Browner WS. Casefinding instruments for depression. Two questions are as good as many. Journal of General Internal Medicine 1997; 12: 439-45.

4. National Institute for Clinical Excellence Depression: management of depression in primary and secondary care. London. National Institute for Clinical Excellence, 2004.

5. Taylor D, Paton C, Kerwin R, South London and Maudsley NHS Trust, Oxleas NHS Foundation Trust. 2005-2006 Prescribing guidelines. 8th ed. Taylor and Francis, 2005.

Raveen Hanwella and Varuni de Silva, Senior Lecturers, Department of Psychological Medicine, Faculty of Medicine, University of Colombo, Kynsey Road, Colombo 8.

Correspondence: RH, email <raveenhanwella@yahoo.com.uk>. We declare that we have no conflicts of interest in regard to this article.

\section{Care of the mind}

The care of the human mind is the most noble branch of medicine.

Aloysius Sieffert. Medical and Surgical Practitioners' Memorandum (circa 1858). 